\title{
Palbociclib: a first-in-class CDK4/CDK6 inhibitor for the treatment of hormone-receptor positive advanced breast cancer
}

Janice Lu

\begin{abstract}
Palbociclib was approved by the FDA for use in combination with letrozole for the treatment of postmenopausal women with hormone-receptor-positive, HER2-negative advanced breast cancer as initial endocrine-based therapy. In addition, the combination of palbociclib with fulvestrant resulted in superior outcome than fulvestrant alone in those who had progressed during prior endocrine therapy. This research highlight summarized the current development of CDK4/CDK6 inhibitors and future directions in the treatment of advanced hormone-receptor-positive breast cancer.
\end{abstract}

Keywords: Breast cancer, CDK4/CDK6 inhibitor, Palbociclib

Personalizing the use of cancer therapeutics is a major focus of current cancer research [1-6]. The pioneering work of Finn and Slamon showed activity of palbociclib as an inhibitor of cyclin-dependent kinase (CDK) 4 and 6 which reduced cellular proliferation of estrogen receptor (ER)-positive breast cancer cell lines by blocking progression of cells from G1 into S phase of the cell cycle [7]. Palbociclib was approved by the FDA for use in combination with letrozole for the treatment of postmenopausal women with ER-positive, human epidermal growth factor receptor 2 (HER2)-negative advanced breast cancer as initial endocrine-based therapy for their metastatic disease.

The approval of palbociclib is based on a phase 2 PALOMA-1/TRIO-18 study, which is a randomized, multicenter, open-label trial in postmenopausal women with HR-positive, HER2-negative, advanced breast cancer who had not received previous systemic treatment for advanced disease. The trial enrolled 165 patients randomly allocated to receive either palbociclib plus letrozole or letrozole alone [8]. Among the 165 patients, $43 \%$ had received chemotherapy and $33 \%$ had received anti-hormonal therapy as a neoadjuvant or adjuvant treatment. Forty-nine percent of patients had no prior

Correspondence: mjlu@mednet.ucla.edu

Division of Hematology/Oncology, Department of Medicine, David Geffen School of Medicine, University of California, Los Angeles, CA, USA systemic therapy in the neoadjuvant or adjuvant setting. The majority of patients (98\%) had metastatic disease, $48 \%$ had visceral disease, $75 \%$ had bone disease, and $19 \%$ had bone-only disease. Median progression-free survival was 10.2 months (95\% CI 5.7-12.6) for the letrozole group and 20.2 months (13.8-27.5) for the palbociclib plus letrozole group (HR 0.488, $95 \% \mathrm{CI}$ $0.319-0.748$; one-sided $p=0.0004$ ), which correlates to a doubled PFS in favor of the combination group. The overall survival is unknown and the follow-up is ongoing.

In addition to the benefit of palbociclib as initial endocrine-based therapy for metastatic hormone-receptorpositive breast cancer, PALOMA3 trial evaluated 521 patients with advanced hormone-receptor-positive, HER2-negative advanced breast cancer that had relapsed or progressed during prior endocrine therapy. Patients were randomly assigned in a 2:1 ratio to receive palbociclib and fulvestrant or placebo and fulvestrant [9]. PALOMA3 study concluded that palbociclib with fulvestrant resulted in longer progression-free survival and a relatively higher quality of life than fulvestrant alone in patients with advanced hormone-receptorpositive breast cancer that had progressed during prior endocrine therapy. The median progression-free survival was 9.2 months with palbociclib-fulvestrant and 3.8 months with placebo-fulvestrant $(p<0.0001,95 \% \mathrm{CI})$. 
Consistent benefit from palbociclib was seen in all subgroups analyzed, with similar benefit in progressionfree survival adding palbociclib in both premenopausal and postmenopausal women. Translational research for markers that might predict which group of patients benefits most is ongoing.

In addition to the favorable outcome from the clinical trials using palbociclib, other small molecule inhibitors of CDK $4 / 6$ are being studied in hope for developing more potent agents. In preclinical models, LEE011 (ribociclib) has demonstrated a dose-dependent antitumor activity that tracks well with CDK4/6 inhibition $[10,11]$. Ribociclib is currently being evaluated in HRpositive breast cancer with letrozole and PI3K inhibitor BYL719 $[12,13]$. A phase III study evaluating the combination of ribociclib with letrozole in HR-positive, HER2-negative breast cancer is ongoing (MONALESSA-2, NCT01958021). Other phase III studies investigating ribociclib in combination regimens for the treatment of women with HR-positive, HER2-negative advanced breast cancer are ongoing (MONALEESA-3, NCT02422615; [14]).

Abemaciclib (LY2835219) is another selective oral CDK4/6 inhibitor that is being developed in preclinical settings and clinical trials [15-17]. The combination of abemaciclib plus fulvestrant was evaluated in a small study $(n=13)$, which showed that combination therapy was well tolerated [18]. Several studies are planned which include a phase III randomized double-blind placebo-controlled trial of nonsteroidal aromatase inhibitor with or without abemaciclib in previously untreated advanced hormone-sensitive breast cancer (MONARCH 3, NCT02246621) [19]. A randomized double-blind placebocontrolled phase III study will compare the combination of abemaciclib with fulvestrant versus fulvestrant alone (MONARCH 2, NCT02107703) to investigate the benefit of abemaciclib in combination with endocrine therapy with a primary endpoint of PFS.

Future directions also include using the CDK4 and CDK6 inhibitors in the adjuvant and neoadjuvant therapy settings. A neoadjuvant trial investigating the combination of abemaciclib and aromatase inhibitor in locally advanced ER-positive, HER2-negative breast cancer (neoMONARCH, NCT02441946) is ongoing.

The results of the PALOMA $1 /$ TRIO 18 study led to accelerated FDA approval of palbociclib in combination with letrozole in first line advanced ER+/HER2-breast cancer. The doubled PFS benefit observed in this randomized trial was impressive, but it still is awaiting confirmation from the phase III PALOMA-2 study. The PALOMA 3 study clearly showed that adding palbociclib to fulvestrant resulted in substantially longer progressionfree survival than fulvestrant alone in patients with advanced HR-positive and HER2-negative breast cancer that had progressed during prior endocrine therapy, regardless of menopause status. In addition, the combination therapy is associated with relatively higher quality of life than with fulvestrant alone. Neutropenia was the most common adverse event in patients receiving palbociclib with low incidence of febrile neutropenia in both treatment groups. Although the combination of aromatase inhibitor with fulvestrant or everolimus remain excellent treatments for this group of patients [1, 20], PALOMA 3 offers an excellent alternative therapy for both premenopausal and postmenopausal patients who had progressed with prior endocrine therapy. Palbociclib and other CDK4/6 inhibitors being developed offer a new era of treatment for hormone-receptor-positive advanced breast cancer.

\section{Competing interests}

The author declares that she has no competing interests.

\section{Acknowledgements}

This study was supported by the Research Foundation at UCLA.

Received: 30 June 2015 Accepted: 27 July 2015

Published online: 13 August 2015

\section{References}

1. Baselga J, Campone M, Piccart M, Burris 3rd HA, Rugo HS, Sahmoud T, et al. Everolimus in postmenopausal hormone-receptor-positive advanced breast cancer. N Engl J Med. 2012;366(6):520-9.

2. Akinleye A, Avvaru P, Furqan M, Song Y, Liu D. Phosphatidylinositol 3-kinase (PI3K) inhibitors as cancer therapeutics. J Hematol Oncol. 2013;6(1):88.

3. Takalkar A, Adams S, Subbiah V. Radium-223 dichloride bone-targeted alpha particle therapy for hormone-refractory breast cancer metastatic to bone. Experimental Hematol Oncol. 2014;3:23.

4. Verma S, Miles D, Gianni L, Krop IE, Welslau M, Baselga J, et al. Trastuzumab emtansine for HER2-positive advanced breast cancer. N Engl J Med. 2012;367(19):1783-91.

5. Incorvati JA, Shah S, Mu Y, Lu J. Targeted therapy for HER2 positive breast cancer. J Hematol Oncol. 2013;6:38.

6. Akinleye A, Chen Y, Mukhi N, Song Y, Liu D. Ibrutinib and novel BTK inhibitors in clinical development. J Hematol Oncol. 2013;6(1):59.

7. Finn R, Dering J, Conklin D, Kalous O, Cohen D, Desai A, et al. PD 0332991, a selective cyclin D kinase 4/6 inhibitor, preferentially inhibits proliferation of luminal estrogen receptor-positive human breast cancer cell lines in vitro. Breast Cancer Res. 2009;11(5):R77.

8. Finn RS, Crown JP, Lang I, Boer K, Bondarenko IM, Kulyk SO, et al. The cyclin-dependent kinase $4 / 6$ inhibitor palbociclib in combination with letrozole versus letrozole alone as first-line treatment of oestrogen receptor-positive, HER2-negative, advanced breast cancer (PALOMA-1/ TRIO-18): a randomised phase 2 study. Lancet Oncol. 2015;16(1):25-35.

9. Turner NC, Ro J, Andre F, Loi S, Verma S, Iwata H, Harbeck N, Loibl S, Huang Bartlett C, Zhang K, Giorgetti C, Randolph S, Koehler M, Cristofanilli M: Palbociclib in hormone-receptor-positive advanced breast cancer. The New England journal of medicine 2015(June 1): doi: 10.1056/NEJMoa1505270.

10. Kim S, Loo A, Chopra R, Caponigro G, Huang A, Vora S, et al. Abstract PRO2: LEE011: an orally bioavailable, selective small molecule inhibitor of CDK4/ 6-reactivating Rb in cancer. Mol Cancer Ther. 2013;12(11 Supplement):R02.

11. Zhang Y-X, Sicinska E, Czaplinski JT, Remillard SP, Moss S, Wang Y, et al. Antiproliferative effects of CDK4/6 inhibition in CDK4-amplified human liposarcoma in vitro and in vivo. Mol Cancer Ther. 2014;13(9):2184-93.

12. Munster PN, Hamilton EP, Estevez LG, De Boer RH, Mayer IA, Campone M, et al. Ph IB study of LEE011 and BYL719 in combination with letrozole in ER+, HER2-breast cancer. ASCO Meeting Abstracts. 2014;32(26_suppl):143.

13. Munster PN, Hamilton EP, Franklin C, Bhansali S, Wan K, Hewes B, et al. Phase Ib study of LEE011 and BYL719 in combination with letrozole in estrogen receptor-positive, HER2-negative breast cancer (ER+, HER2- BC). ASCO Meeting Abstracts. 2014;32(15_suppl):533. 
14. Tripathy D, Bardia A, Hurvitz SA, Harbeck N, Colleoni M, Franke FA, et al. Phase III, randomized, double-blind, placebo-controlled study of ribociclib (LEE011) in combination with either tamoxifen and goserelin or a non-steroidal aromatase inhibitor (NSAI) and goserelin for the treatment of premenopausal women with HR+, HER2-advanced breast cancer (aBC): MONALEESA-7. ASCO Meeting Abstracts. 2015;33(15 suppl):TPS625.

15. Gelbert LM, Cai S, Lin X, Sanchez-Martinez C, Del Prado M, Lallena MJ, et al. Preclinical characterization of the CDK4/6 inhibitor LY2835219: in-vivo cell cycle-dependent/independent anti-tumor activities alone/in combination with gemcitabine. Investig New Drugs. 2014;32(5):825-37.

16. Tate SC, Cai S, Ajamie RT, Burke T, Beckmann RP, Chan EM, et al. Semi-mechanistic pharmacokinetic/pharmacodynamic modeling of the antitumor activity of LY2835219, a new cyclin-dependent kinase 4/6 inhibitor, in mice bearing human tumor xenografts. Clin Cancer Res. 2014;20(14):3763-74.

17. Shapiro G, Rosen LS, Tolcher AW, Goldman JW, Gandhi L, Papadopoulos KP, et al. A first-in-human phase I study of the CDK4/6 inhibitor, LY2835219, for patients with advanced cancer. ASCO Meeting Abstracts. 2013;31(15_suppl):2500

18. Patnaik A, Rosen LS, Tolaney SM, Tolcher AW, Goldman JW, Gandhi L, et al. LY2835219, a novel cell cycle inhibitor selective for CDK4/6, in combination with fulvestrant for patients with hormone receptor positive (HR+) metastatic breast cancer. ASCO Meeting Abstracts. 2014;32(15_suppl):534.

19. Goetz MP, Toi M, Klise S, Frenzel M, Bourayou N, Di Leo A. MONARCH 3: a randomized phase III study of anastrozole or letrozole plus abemaciclib, a CDK4/6 inhibitor, or placebo in first-line treatment of women with HR+, HER2-locoregionally recurrent or metastatic breast cancer (MBC). ASCO Meeting Abstracts. 2015;33(15_suppl):TPS624.

20. Mehta RS, Barlow WE, Albain KS, Vandenberg TA, Dakhil SR, Tirumali NR, et al. Combination anastrozole and fulvestrant in metastatic breast cancer. N Engl J Med. 2012;367(5):435-44.

\section{Submit your next manuscript to BioMed Central and take full advantage of:}

- Convenient online submission

- Thorough peer review

- No space constraints or color figure charges

- Immediate publication on acceptance

- Inclusion in PubMed, CAS, Scopus and Google Scholar

- Research which is freely available for redistribution 